\title{
Neoadjuvant Chemotherapy for Clinical Stage II and III Thoracic Esophageal Squamous Cell Carcinoma with Curative Esophagectomy
}

\author{
Masahide Ikeguchi ${ }^{*}$, Yusuke Kohno ${ }^{1}$, Kyoichi Kihara', Kazunori Suzuki ${ }^{1}$, \\ Kanenori Endo1, Seiichi Nakamura1, Takashi Sawada1, Tetsu Shimizu1, \\ Tomoyuki Matsunaga'2, Yoji Fukumoto², Hiroaki Saito \\ ${ }^{1}$ Tottori Prefectural Central Hospital, Tottori, Japan \\ ${ }^{2}$ Department of Surgery, Division of Surgical Oncology, Faculty of Medicine, Tottori University, Tottori, Japan \\ Email: ${ }^{*}$ ikeguchim@pref.tottori.jp
}

Received 10 November 2015; accepted 8 December 2015; published 11 December 2015

Copyright (C) 2015 by authors and Scientific Research Publishing Inc.

This work is licensed under the Creative Commons Attribution International License (CC BY). http://creativecommons.org/licenses/by/4.0/

(c) (i) Open Access

\section{Abstract}

Esophageal squamous cell carcinoma (ESCC) is a gastrointestinal carcinoma with a poor prognosis. To improve the survival of patients with this disease, neoadjuvant chemotherapy (NAC) has been introduced. However, the survival benefits of NAC or the correlation between NAC and postoperative complications have not been well considered. In the present study, we retrospectively investigated the clinicopathological effectiveness of NAC in patients with clinical stage II and III thoracic ESCC. This retrospective study enrolled 63 patients with clinical stage II and III thoracic ESCC, who underwent resection of the thoracic esophagus and three-field lymph node dissection between January 2007 and December 2013. NAC with cisplatin plus 5-fluorouracil (5-FU) was introduced in 38 patients. NAC did not correlate with the occurrence of postoperative complications. The 5-year disease-free survival (DFS) rate of the 38 patients with NAC $(41.6 \%)$ was similar to that for the 25 patients who did not receive NAC $(38.1 \%$; $P=0.784)$. However, we found that the DFS of 17 patients with histopathological Grade 2 and 3 tumors who received NAC (5-year DFS rate: 58.1\%) was significantly higher than that of 21 patients with low histopathological grade tumors who received NAC (5-year DFS rate: $28.6 \%$ ), or than that of the 25 patients who did not receive NAC (38.1\%). Moreover, we found that the effectiveness of NAC assessed macroscopically did not correlate with the effectiveness of NAC assessed microscopically. These findings may indicate that preoperative estimation of NAC effectiveness is important in avoiding unnecessary adverse drug effects caused by NAC, and in prolonging the survival of patients with thoracic ESCC.

\footnotetext{
${ }^{*}$ Corresponding author.

How to cite this paper: Ikeguchi, M., Kohno, Y., Kihara, K., Suzuki, K., Endo, K., Nakamura, S., Sawada, T., Shimizu, T., Matsunaga, T., Fukumoto, Y. and Saito, H. (2015) Neoadjuvant Chemotherapy for Clinical Stage II and III Thoracic Esophageal Squamous Cell Carcinoma with Curative Esophagectomy. Journal of Cancer Therapy, 6, 1207-1213. http://dx.doi.org/10.4236/jct.2015.615131
} 


\section{Keywords}

\section{Esophageal Squamous Cell Carcinoma, Histopathological Grade, Neoadjuvant Chemotherapy, Prognosis}

\section{Introduction}

Studies on the distribution of lymph-node metastases in patients with resected thoracic esophageal squamous cell carcinoma (ESCC) have revealed extensive metastases to lymph nodes located in the neck, chest and abdomen [1]. Thus, in Japan, subtotal esophagectomy with radical three-field dissection of cervical, thoracic and abdominal lymph nodes has been introduced as an effective therapeutic strategy for localized thoracic ESCC [2]. However, the prognosis of patients with thoracic ESCC remains poor and many patients die as a result of tumor recurrence, even after curative resection has been performed.

The Japan Clinical Oncology Group (JCOG) has conducted two randomized controlled trials (JCOG 9204 and the JCOG 9907) to assess the potential benefits of adding adjuvant therapy to surgery in patients with ESCC [3] [4]. The JCOG 9204 study assessed the benefit of postoperative adjuvant chemotherapy with cisplatin plus 5 -fluorouracil (5-FU) as compared with surgery alone in patients with resectable stage I or II esophageal cancer. Overall survival did not differ significantly between the groups (5-year survival rate, 52\% of surgery alone group vs. $61 \%$ of surgery plus adjuvant chemotherapy group; $P=0.13$ ). Disease-free survival was improved significantly in the patients who received postoperative chemotherapy, and especially in patients with lymph node metastasis. In the JCOG 9907 study, neoadjuvant chemotherapy (NAC) with cisplatin plus 5-FU was compared with postoperative chemotherapy with cisplatin plus 5-FU in patients with clinical stage II or III esophageal cancer. NAC was found to be superior to postoperative chemotherapy in terms of overall survival. The 5 -year survival rate was $60 \%$ in the neoadjuvant group and $38 \%$ in the adjuvant group $(P=0.013)$. On the basis of these results, NAC followed by radical surgery is recommended in cases of locally advanced squamous cell carcinoma.

However, the influence of NAC on postoperative complications has not been well discussed. In the present study, we retrospectively compared mortality and morbidity after surgery between the two groups (the group treated with NAC and the group not treated with NAC) consisting of clinical stage II and III patients with operable thoracic ESCC.

\section{Materials and Methods}

\subsection{Patients}

Esophagogastroscopy, computed tomography (CT) scanning, endoscopic ultrasound and positron emission topography were all routinely used in staging the tumors of patients diagnosed with ESCC. Fitness for surgery was assessed through clinical examination, pulmonary function tests, electrocardiography, echocardiography and exercise tolerance testing. After physical examination, and hematological and biochemical investigations, patients with potentially resectable disease who were deemed physiologically fit for surgery were analyzed in this study. Patients with T4 cancer (invasion to adjacent organs), with distant metastasis (to lung, liver, bone or extended lymph nodes), with a poor Eastern Cooperative Oncology Group Performance Status (PS $\geq 2$ ), with sever dysfunction of organs (such as cardio-pulmonary, liver and kidney), of advanced age ( $>80$ years) were excluded from surgical treatments in our institute. Between January 2007 and December 2013, 63 patients with clinical stage II and III thoracic ESCC underwent resection of the thoracic esophagus and three-field lymph node dissection at Tottori University Hospital. Patients who underwent transhiatal esophagectomy without thoracotomy, left side thoracotomy with laparotomy plus two-field lymph node dissection, and a salvage operation were excluded from this study. The mean age of patients was 65.4 years (range, 49 - 78 years). Fifty-six were males and seven were females. All patients were followed up at Tottori University Hospital until September 2015. The median follow-up period was 32 months (range, 2 - 101 months). Written informed consent was required from all patients before treatment. All investigations reported in this study were conducted in conformity with the Recommendations from the Declaration of Helsinki. 


\subsection{Surgical Procedures}

Between January 2007 and August 2009, traditional right antero-lateral thoracotomy with laparotomy (open method) has been routinely performed. Since September 2009, thoracoscopic thoracic esophagectomy with patients in the prone position, has been undertaken (thoracoscopic method). However, right antero-lateral thoracotomy was indicated for cases involving strong adhesion between the lung and the pleura. Twenty-six patients were treated using the open method and 37 using the thoracoscopic method. In both methods, intraoperative histological analysis of frozen-sections of tissue from the proximal margin was routinely performed. The stomach was delivered into the thorax and a $5 \mathrm{~cm}$ circumference gastric tube fashioned using a stapler was inserted in 54 patients. However, in nine patients with double cancers (esophageal and gastric cancer) or gastrectomized cases, the jejunum was elongated to the cervical esophagus or pharynx through the ante-sternum root. Anastomoses between the cervical esophagus or pharynx and the gastric tube or jejunum were performed using the Gambee method.

\subsection{Chemotherapy}

S-1 adjuvant chemotherapy has routinely been introduced for patients with stage II or III ESCC in our institute. Since 2010, in accordance with the results from the JCOG 9204 and 9907 studies, NAC involving cisplatin plus 5-FU has been offered to patients who had been diagnosed as having clinical stage II and III thoracic ESCC in our institute. One course of chemotherapy was delivered in 3 weeks. The patients underwent two courses of protracted infusion of 5-FU ( $800 \mathrm{mg} / \mathrm{m}^{2} /$ day) on days 1 - 4 and days 29 - 32, and 2-h infusions of cisplatin on days 1 and $29\left(80 \mathrm{mg} / \mathrm{m}^{2}\right)$, together with adequate hydration and antiemetic coverage. Surgical resection was carried out at 6 - 8 weeks after the completion of chemotherapy. However, if the patients did not agree with NAC treatment, traditional S-1 adjuvant chemotherapy was introduced. Thus, in 63 patients, NAC was planned for 38 patients and adjuvant chemotherapy was planned for 25 patients.

\subsection{Macroscopic Evaluation of the Effectiveness of NAC}

In cases of NAC, we evaluated the effectiveness of NAC by comparing the findings from endoscopy and CT before and after NAC. Treatment effectiveness was determined in accordance with the guidelines of the Response Evaluation Criteria in Solid Tumors (RECIST) Group [5].

\subsection{Pathological Analysis}

The specimens together with any separately harvested lymph nodes were classified using the Union for International Cancer Control (UICC) TNM classification for esophageal cancer [6]. An R0 resection was defined as complete tumor excision with all margins histologically free of tumor. In accordance with the UICC TNM classification, histopathological stage I thoracic ESCC was detected in 12 patients, stage II in 19 and stage III in 32. The effectiveness of NAC was determined histopathologically in accordance with the grading system of the Japanese Classification of Esophageal Carcinoma [7]. Grading was as follows: Grade 0, no part of the tumor was affected; Grade 1, less than two-thirds of the tumor was affected; Grade 2, between two-thirds and the entire tumor was affected; and Grade 3, no residual tumor was present (pathologically complete response).

\subsection{Statistical Analysis}

The Mann-Whitney $U$ test, Chi-squared test and Fisher's exact probability test were used to compare the clinicopathological characteristics of the two groups. Long-term survival was calculated using the Kaplan-Meier method and the prognostic differences between the two groups was compared using the log-rank test. A $P$-value $<0.05$ was considered statistically significant.

\section{Results}

A total of 63 patients diagnosed as having clinical stage II and III thoracic ESCC who were considered fit for a surgical treatment strategy underwent esophagectomy, and R0 operations were performed. A total of 37 of these patients were treated using the thoracoscopic method while 26 underwent the open method. Esophageal substitution involving a gastric tube was used in 54 patients and the jejunum was used in nine patients. The clinical 
characteristics of the 63 patients are detailed in Table 1. NAC with cisplatin plus 5-FU was introduced in 38 patients.

Surgical complications were evident in 29 (46\%) patients. Anastomotic leakage occurred in 20 patients, chylothorax in four, pneumonia in three and necrosis of the substituted esophagus in two. One patient with postoperative chylothorax died within 2 months after surgery. Thus, the surgical morbidity was $46 \%$ and the mortality was $1.6 \%$. The mean postoperative hospital stay of the 29 patients with postoperative complications (87.4 days) was significantly longer than that of the 34 patients who did not have complications (27.6 days; $P<0.001$ ). The correlations regarding the occurrence of postoperative complications and clinical factors were analyzed (Table 2). Median values were used as cut-off values for patient age, operation time, intraoperative blood loss and number of dissected lymph nodes. We found that NAC was not a factor in postoperative complications.

Table 1. Clinical characteristics of the 63 patients with clinical stage II and III thoracic esophageal squamous cell carcinoma.

\begin{tabular}{lc}
\hline & Clinical data \\
\hline Age (Mean, median, range, years) & $65.4(66,49-78)$ \\
Gender (Male/Female) & $56 / 7$ \\
Operation time (Mean, median, range, minutes) & $657.9(644,420-984)$ \\
Intraoperative blood loss (Mean, median, range, mL) & $519.9(330,10-3020)$ \\
Number of dissected lymph nodes (Mean, median, range) & $51.4(52,21-105)$ \\
Clinical tumor stage (II/III) & $25 / 38$ \\
Histological tumor stage (I/II/III) & $12 / 19 / 32$ \\
Postoperative complications (Yes/No) & $29 / 34$ \\
Postoperative hospital stay (Mean, median, range, days) & $55.1(32,10-478)$
\end{tabular}

Table 2. The correlations regarding the occurrence of postoperative complications and clinical factors.

\begin{tabular}{|c|c|c|c|}
\hline & $\mathrm{N}$ & Occurrence of postoperative complication & $P$ \\
\hline \multicolumn{4}{|l|}{ Age (years) } \\
\hline$\geq 66$ & 34 & $16(47.1 \%)$ & 0.859 \\
\hline$<66$ & 29 & $13(44.8 \%)$ & \\
\hline \multicolumn{4}{|l|}{ Operation time (minutes) } \\
\hline$\geq 644$ & 32 & $16(50 \%)$ & 0.521 \\
\hline$<644$ & 31 & $13(41.9 \%)$ & \\
\hline \multicolumn{4}{|c|}{ Intraoperative blood loss (mL) } \\
\hline$\geq 330$ & 32 & $18(56.3 \%)$ & 0.098 \\
\hline$<330$ & 31 & $11(35.5 \%)$ & \\
\hline \multicolumn{4}{|c|}{ Number of dissected lymph nodes } \\
\hline$\geq 52$ & 32 & $13(40.6 \%)$ & 0.382 \\
\hline$<52$ & 31 & $16(51.6 \%)$ & \\
\hline \multicolumn{4}{|l|}{ Clinical stage } \\
\hline II & 25 & $10(40 \%)$ & 0.439 \\
\hline III & 38 & $19(50 \%)$ & \\
\hline \multicolumn{4}{|l|}{ Surgery } \\
\hline Open method & 26 & $15(57.7 \%)$ & 0.12 \\
\hline Thoracoscopic method & 37 & $14(37.8 \%)$ & \\
\hline \multicolumn{4}{|l|}{ NAC } \\
\hline Yes & 38 & $15(39.5 \%)$ & 0.198 \\
\hline No & 25 & $14(56 \%)$ & \\
\hline
\end{tabular}

NAC: neoadjuvant chemotherapy. 
The 5-year disease-free survival (DFS) rate and 5-year overall survival (OS) rate of all patients were $40.7 \%$ and $44.7 \%$, respectively. In the 38 patients treated with NAC, only one course was performed in six patients (15.8\%) because of severe adverse events (neutropenia, 4; diarrhea, 1 ; renal dysfunction, 1); two courses of NAC was accomplished in the remaining 32 patients. Adjuvant chemotherapy using S-1 was planned for 25 patients. However, because of surgical complications and declining patient PS, S-1 adjuvant chemotherapy could be administered to only 7/25 (28\%) of the patients. The long-term outcomes were compared. The 5-year DFS and 5-year OS of the 38 patients treated with NAC (41.6\% and 45\%, respectively) were not significantly different from those of the 25 patients who were not treated with NAC (38.1\% and $44.2 \%$, respectively; $P=0.784$ and $P=0.855$, respectively). In addition, the 5-year DFS and 5-year OS of the 34 patients who did not have postoperative complications ( $40.3 \%$ and $48.6 \%$, respectively) were similar to those of the 29 patients with postoperative complications ( $42.5 \%$ and $39.4 \%$, respectively; $P=0.873$ and $P=0.221$, respectively).

In the 38 patients treated with NAC, a partial response (PR) was detected in nine (23.7\%) at preoperative evaluation of NAC according to the RECIST criteria. Stable disease (SD) was achieved in 26 patients and progressive disease (PD) in three. The 5-year DFS and the 5-year OS of the nine patients with a PR (50\% and $27.8 \%$, respectively) were not significantly different from those of the 26 patients with an SD and the three patients with a PD (39.5\% and 50.6\%, respectively; $P=0.956$ and $P=0.496$, respectively). In addition, histopathological Grade 0 disease was detected in one patient, Grade 1 in 20, Grade 2 in 16 and Grade 3 in one. Twenty-one patients with histopathological Grade 0 and 1 disease were divided into the low grade group and 17 patients with histopathological Grade 2 and 3 disease were divided into the high grade group, respectively. No correlation was detected between the RECIST criteria and histopathological grading $(P=0.984)$. The 5-year DFS rate of the 17 patients in the histopathological high grade group (58.1\%) was significantly higher than that of the 21 patients in the histopathological low grade group (28.6\%; $P=0.048$; Figure 1). However, the 5-year OS of both group was not different (high grade group, $56.5 \%$; low grade group, $37.4 \% ; P=0.218$ ). We found no correlation between the successful execution of NAC treatment and histopathological effectiveness $(P=0.132$; data not shown), and no correlation between successful execution of NAC treatment and DFS $(P=0.652$; data not shown).

\section{Discussion}

In this retrospective study, we found that NAC did not correlate with the development of postoperative complications such as anastomotic leakage, respiratory complications (defined as isolated respiratory compromise secondary to pneumonia, persistent pneumothorax or pulmonary embolism), cardiovascular complications (defined as the presence of a persistent arrhythmia or myocardial infarction), chylothorax, wound infections and other infectious complications (including urinary tract infections, line infections and intra-abdominal and mediastinal collections requiring drainage). Hirao et al. [8] and Kumagai et al. [9] reported that preoperative chemotherapy

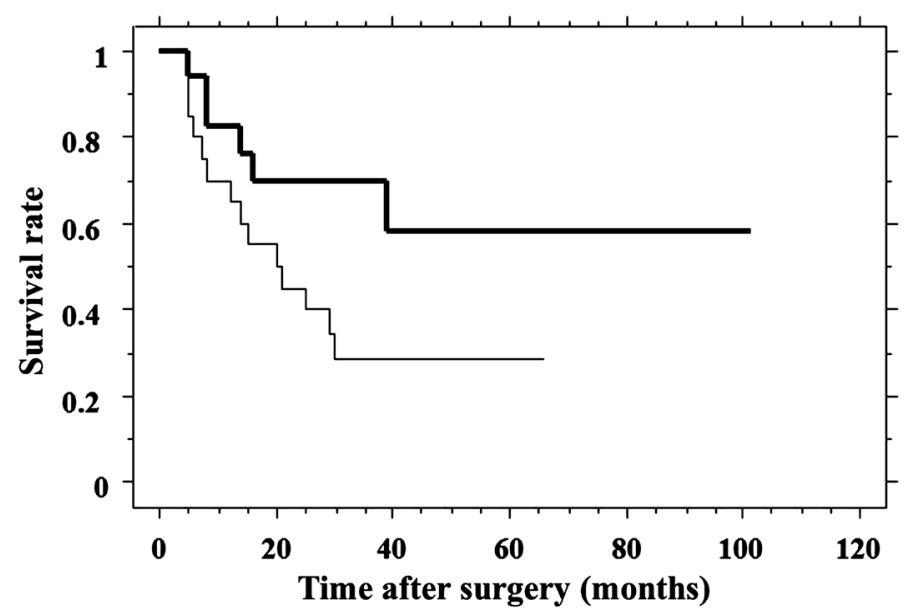

Figure 1. The disease free survival curve for the 17 patients in the histopathological high grade group (thick line) was significantly superior to that for the 21 patients in the histopathological low grade group (thin line; $P=0.048$ ). 
or chemoradiotherapy did not increase the risk of morbidity or mortality. Recent studies have demonstrated that nutritional disadvantage that is evident during NAC may correlate with the occurrence of postoperative complications [10] [11]. Thus, NAC itself may not be one of the causative factors of postoperative complications.

NAC followed by radical esophagectomy prolonged the DFS of patients with locally advanced ESCC but the increase was not significant. In contrast, we found that patients with only high histopathological responses after NAC (Grade 2 - 3; 5-year DFS, 58.1\%) experienced a survival benefit. The 5-year DFS of patients with a low histopathological response after NAC (Grade 0 - 1; 5-year DFS, 28.6\%) was almost at the same low level as that of patients who were not treated with NAC (5-year DFS, 38.1\%). Even the number of patients of our series was small; similar results were reported by Yano et al. [12] and Konishi et al. [13]. These findings strongly indicate that a good histopathological response to anticancer drugs may be the key for achieving a favorable prognosis in patients with advanced ESCC. We found estrangement between the macroscopic chemotherapeutic effectiveness determined using endoscopy or CT and histopathological chemotherapeutic effectiveness in our series. Thickened fibrotic areas and muscle disarray caused by fibrosis after chemotherapy might be one of the factors responsible for the discrepancy between the macroscopic and histopathologic decisions regarding NAC efficacy [14]. Correct preoperative estimation of NAC is important. If it is decided that the cisplatin plus 5-FU regimen is not effective, it is necessary to change the NAC regimen to another anticancer drug preoperatively, such as docetaxel [15]. Elliott et al. [16] reported that changes in fluorodeoxyglucose uptake and the maximum standardized uptake value of tumors, detected using positron emission tomography with CT, were useful parameters for the prediction of the histological effectiveness of NAC. Additionally, Makuuchi et al. [17] reported that an increased serum level of soluble interleukin-6 receptor predicted a poor histopathological response to NAC, and poor survival in patients with ESCC. Thus, to avoid unnecessary adverse drug effects caused by NAC and to prolong the survival of patients with thoracic ESCC, it is important to elucidate the effective markers of histopathological changes during NAC.

\section{References}

[1] Ando, N., Ozawa, S., Kitagawa, Y., Shinozawa, Y. and Kitajima, M. (2000) Improvement in the Results of Surgical Treatment of Advanced Squamous Esophageal Carcinoma during 15 Consecutive Years. Annals of Surgery, 232, 225232. http://dx.doi.org/10.1097/00000658-200008000-00013

[2] Akiyama, H., Tsurumaru, M., Udagawa, H. and Kajiyama, Y. (1994) Radical Lymph Node Dissection for Cancer of the Thoracic Esophagus. Annals of Surgery, 220, 364-372. http://dx.doi.org/10.1097/00000658-199409000-00012

[3] Ando, N., Iizuka, T., Ide, H., Ishida, K., Shinoda, M., Nishimaki, T., Takiyama, W., Watanabe, H., Isono, K., Aoyama, N., Makuuchi, H., Tanaka, O., Yamana, H., Ikeuchi, T., Nagai, K., Shimada, Y., Kinjo, Y., Fukuda, H. and Japan Clinical Oncology Group (2003) Surgery plus Chemotherapy Compared with Surgery Alone for Localized Squamous Cell Carcinoma of the Thoracic Esophagus: A Japan Clinical Oncology Group Study_JCOG9204. Journal of Clinical Oncology, 21, 4592-4596. http://dx.doi.org/10.1200/JCO.2003.12.095

[4] Ando, N., Kato, H., Igaki, H., Shinoda, M., Ozawa, S., Shimizu, H., Nakamura, T., Yabusaki, H., Aoyama, N., Kurita, A., Ikeda, K., Kanda, T., Tsujinaka, T., Nakamura, K. and Fukuda, H. (2012) A Randomized Trial Comparing Postoperative Adjuvant Chemotherapy with Cisplatin and 5-Fluorouracil versus Preoperative Chemotherapy for Localized Advanced Squamous Cell Carcinoma of the Thoracic Esophagus (JCOG9907). Annals of Surgical Oncology, 19, 6874. http://dx.doi.org/10.1245/s10434-011-2049-9

[5] Therasse, P., Arbuck, S.G., Eisenhauer, E.A., Wanders, J., Kaplan, R.S., Rubinstein, L., Verweij, J., Glabbeke, M.V., van Oosterom, A.T., Christian, M.C. and Gwyther, S.G. (2000) New Guidelines to Evaluate the Response to Treatment in Solid Tumors. JNCI, 92, 205-216. http://dx.doi.org/10.1093/jnci/92.3.205

[6] Sobin, L.H. and Wittekind, C.H. (2002) UICC TNM Classification of Malignant Tumours. 6th Edition, John Wiley \& Sons, Hoboken, New Jersey.

[7] Japanese Society for Esophageal Diseases (2004) Guidelines for Clinical and Pathologic Studies on Carcinoma of the Esophagus Ninth Edition: Part II. Esophagus, 1, 107-125. http://dx.doi.org/10.1007/s10388-004-0019-z

[8] Hirao, M., Ando, N., Tshujinaka, T., Udagawa, H., Yano, M., Yamana, H., Nagai, K., Mizusawa, J., Nakamura, K. and Japanese Esophageal Oncology Group/Japan Clinical Oncology Group (2011) Influence of Preoperative Chemotherapy for Advanced Thoracic Oesophageal Squamous Cell Carcinoma on Perioperative Complications. British Journal of Surgery, 98, 1735-1741. http://dx.doi.org/10.1002/bjs.7683

[9] Kumagai, K., Rouvelas, I., Tsai, J.A., Mariosa, D., Klevebro, F., Lindblad, M., Ye, W., Lundell, L. and Nisson, M. (2014) Meta-Analysis of Postoperative Morbidity and Perioperative Mortality in Patients Receiving Neoadjuvant Chemotherapy or Chemoradiotherapy for Resectable Oesophageal and Gastro-Oesophageal Junctional Cancers. British 
Journal of Surgery, 101, 321-338. http://dx.doi.org/10.1002/bjs.9418

[10] Yip, C., Goh, V., Davies, A., Gossage, J., Mitchell-Hay, R., Hynes, O., Maisey, N., Ross, P., Gaya, A., Landau, D.B., Cook, G.J., Griffin, N. and Mason, R. (2014) Assessment of Sarcopenia and Changes in Body Composition after Neoadjuvant Chemotherapy and Associations with Clinical Outcomes in Oesophageal Cancer. European Radiology, 24, 998-1005. http://dx.doi.org/10.1007/s00330-014-3110-4

[11] Ida, S., Watanabe, M., Karashima, R., Imamura, Y., Ishimoto, T., Baba, Y., Iwagami, S., Sakamoto, Y., Miyamoto, Y., Yoshida, N. and Baba, H. (2014) Changes in Body Composition Secondary to Neoadjuvant Chemotherapy for Advanced Esophageal Cancer Are Related to the Occurrence of Postoperative Complications after Esophagectomy. Annals of Surgical Oncology, 21, 3675- 3679. http://dx.doi.org/10.1245/s10434-014-3737-Z

[12] Yano, M., Takachi, K., Doki, Y., Miyashiro, I., Kishi, K., Noura, S., Eguchi, H., Yamada, T., Ohue, M., Ohigashi, H., Sasaki, Y., Ishikawa, O. and Imaoka, S. (2006) Preoperative Chemotherapy for Clinically Node-Positive Patients with Squamous Cell Carcinoma of the Esophagus. Diseases of the Esophagus, 19, 158-163. http://dx.doi.org/10.1245/s10434-014-3737-z

[13] Konishi, H., Fujiwara, H., Shiozaki, A., Hiramoto, H., Kosuga, T., Komatsu, S., Ichikawa, D., Okamoto, K. and Otsuji, E. (2015) Effects of Neutropenia and Histological Responses in Esophageal Squamous Cell Carcinoma with Neo-Adjuvant Chemotherapy. International Journal of Clinical Oncology, Epub Ahead of Print. http://dx.doi.org/10.1007/s10147-015-0875-7

[14] Lee, C.T., Chow, N.H., Liu, Y.S., Lin, S.C., Lin, P.C., Wu, Y.H., Lee, J.C. and Tsai, H.M. (2012) Computed Tomography with Histological Correlation for Evaluating Tumor Regression of Rectal Carcinoma after Preoperative Chemoradiation Therapy. Hepatogastroenterology, 59, 2484-2489.

[15] Hara, H., Tahara, M., Daiko, H., Kato, K., Igaki, H., Kadowaki, S., Tanaka, Y., Hamamoto, Y., Matsushita, H., Nagase, M. and Hosoya, Y. (2013) Phase II Feasibility Study of Preoperative Chemotherapy with Docetaxel, Cisplatin, and Fluorouracil for Esophageal Squamous Cell Carcinoma. Cancer Science, 104, 1455-1460. http://dx.doi.org/10.1111/cas.12274

[16] Elliott, J.A., O’Farrell, N.J., King, S., Halpenny, D., Malik, V., Muldoon, C., Johnston, C. and Reynolds, J.V. (2014) Value of CT-PET after Neoadjuvant Chemoradiation in the Prediction of Histological Tumour Regression, Nodal Status and Survival in Oesophageal Adenocarcinoma. British Journal of Surgery, 101, 1702-1711. http://dx.doi.org/10.1002/bjs.9670

[17] Makuuchi, Y., Honda, K., Osaka, Y., Kato, K., Kojima, T., Daiko, H., Igaki, H., Ito, Y., Hoshino, S., Tachibana, S., Watanabe, T., Furuta, K., Sekine, S., Umaki, T., Watabe, Y., Miura, N., Ono, M., Tsuchida, A. and Yamada, T. (2013) Soluble Interleukin-6 Receptor Is a Serum Biomarker for the Response of Esophageal Carcinoma to Neoadjuvant Chemoradiotherapy. Cancer Science, 104, 1045-1051. http://dx.doi.org/10.1111/cas.12187 